\title{
The Contribution of the Three-Body Process in the Nonmesonic Weak Decay of the ${ }_{\Lambda}^{12} \mathrm{C}$ Hypernucleus.
}

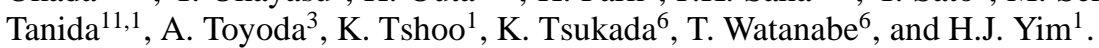 \\ 1 Department of Physics and Astronomy, Seoul National University, Seoul, 151-747, Korea \\ 2 Department of Physics, Osaka University, Toyonaga 560-0043, Japan \\ 3 High Energy Accelerator Research Organization (KEK), Tsukuba 305-0801, Japan \\ 4 Gesellschaft fur Schwerionenforschung mbH (GSI), Darmstadt 64291 Germany \\ 5 Lab. of Physics, Osaka Electro Communication University, Neyagawa 572-8530, Japan \\ 6 Department of Physics, Tohoku University, Sendai 980-8578, Japan \\ 7 Department of Physics, Ewha Womans University, Seoul 120-750, Korea \\ 8 Korea Research Institute of Standards and Science (KRISS), Daejeon 305-600, Korea \\ 9 Department of Physics, University of Tokyo, Hongo 113-0033, Japan \\ 10 Department of Physics, Tokyo Institute of Technology, Ookayama 152-8551, Japan \\ 11 Riken Nishina Center, Riken, Wako 351-0198, Japan \\ 12 Department of Physics, Kyoto University, Kyoto 606-8502, Japan \\ 13 Japan Atomic Energy Research Institute, Tokai 319-1195, Japan
}

H. Bhang ${ }^{1, a}$, S. Ajimura ${ }^{2}$, K. Aoki ${ }^{3}$, A. Banu ${ }^{4}$, T. Fukuda ${ }^{5}$, O. Hashimoto ${ }^{6}$, J.I. Hwang ${ }^{7}$, S. Kameoka ${ }^{6}$, B.H. Kang ${ }^{1}$, E. $\mathrm{Kim}^{1}$, J.H. Kim ${ }^{1,8}$, T. Maruta ${ }^{9}$, Y. Miura ${ }^{6}$, Y. Miyake ${ }^{2}$, T. Nagae ${ }^{3,12}$, M. Nakamura ${ }^{9}$, S.N. Nakamura ${ }^{6}$, H. Noumi $^{3,2}$, S. $^{3}$

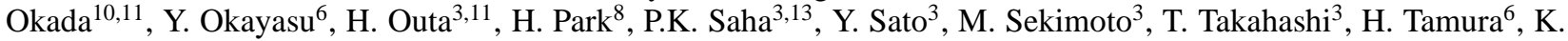

\begin{abstract}
The serious inconsistency problem between the values of experimental and theoretical $\Gamma_{n} / \Gamma_{p}$ ratio of the nonmesonic weak decay of $\Lambda$ hypernuclei has been resolved recently. We have shown that the reason behind the problem was the quenching of the nucleon yields which in turn was due to the contribution of the three-body process. We have measured that the branching ratio of the three-body process in nonmesonic weak decay, $0.29 \pm 0.13$, is so large that the absolute values of $\Gamma_{n}$ and $\Gamma_{p}$ must be determined taking account of the contribution of the 3-body process. In this paper, the recent studies toward the finding of the 3-body process via the exclusive coincidence experiments are presented.
\end{abstract}

\section{Introduction}

A $\Lambda$ hyperon in a nucleus decays via either a mesonic or a nonmesonic weak interaction process. The mesonic weak decay process, $\Lambda \rightarrow \mathrm{N} \pi$, which is the same process as that of free $\Lambda$ is strongly suppressed in the nucleus except in very light nuclei due to the low decay momentum $(\sim 100$ $\mathrm{MeV} / \mathrm{c}$ ). Instead the nonmesonic weak decay (NMWD) channels in which a $\Lambda$ decays interacting with a neighbor nucleon, either a proton or a neutron via $\Lambda \mathrm{p} \rightarrow \mathrm{np}\left(\Gamma_{p}\right)$ or $\Lambda \mathrm{n} \rightarrow \mathrm{nn}\left(\Gamma_{n}\right)$ emitting two energetic high momentum nucleons $(\sim 400 \mathrm{MeV} / \mathrm{c})$ become open and dominant in the nuclei beyond s-shell.

$\Gamma_{n} / \Gamma_{p}$ puzzle: Fundamental interest on NMWD is that it provides practically the only means to study the elementary $\Delta S=1$ baryon-baryon weak interaction process so far since it is very difficult to realize the process in the free space. Among the important issues of NMWD, such as decay widths, asymmetry and the isospin dependence $(\Delta \mathrm{I})$, especially the $\Gamma_{n} / \Gamma_{p}$ ratio has been the long stand-

\footnotetext{
a e-mail: bhang@snu.ac.kr
}

ing concern because of the serious inconsistency between experimental and theoretical values of the ratio. The measured values have been consistently larger than or close to unity while the theoretical ones, both the basic one-pion exchange (OPE) model and the extended models, about 0.1 or so, much smaller than unity [1]. However, the long standing inconsistency has finally been removed in the recent developments. Theoretical ratios have been largely increased from the values around 0.1 which is about the same as that of the elementary OPE model to those distributed in the region of 0.3-0.7, after the correction of the phase of $K$ and $K^{*}$ exchange amplitudes [2-4].

Three-body nonmesonic weak decay process: In an effort to cure the $\Gamma_{n} / \Gamma_{p}$ puzzle problem, in addition to the one-nucleon $(1 \mathrm{~N})$ induced NMWD, the two-nucleon $(2 \mathrm{~N})$ induced NMWD mode in which a $\Lambda$ interacts with a pair nucleons and then three nucleons are emitted in the final states of the decay, namely the $\Lambda \mathrm{NN} \rightarrow \mathrm{nNN}$ process, was first introduced by Alberico et al. in the nuclear matter calculation of the one-meson exchange (OME) models in the referecne [5]. The $2 \mathrm{~N}-\mathrm{NMWD}$ was later applied to finite 


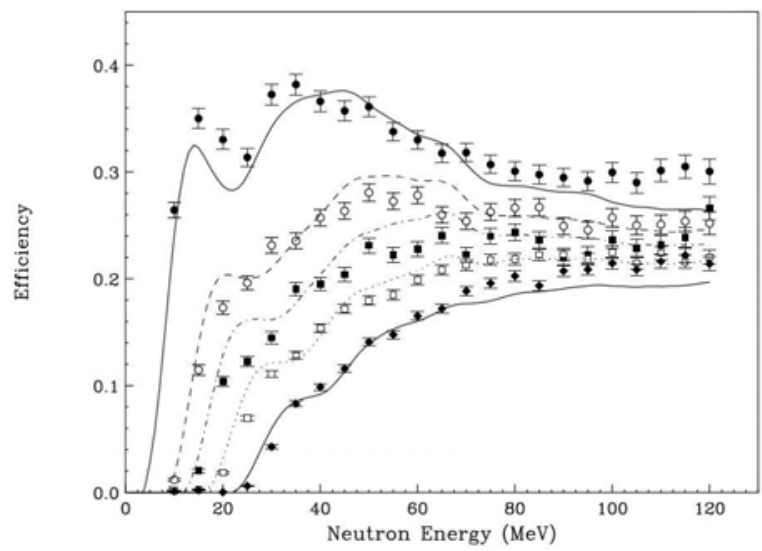

Fig. 1. The neutron detection efficiencies as a function of the neutron energy calculated by the modified DEMONS code (lines), which are compared with those of existing experimental neutron detection efficiencies (data points). The thresholds are 2.80, 5.58, 7.89, 11.15 and 15.75 MeVee from the top [13].

nuclei by Ramos et al. [?]. In the model they calculated the contribution of $2 \mathrm{~N}-\mathrm{NMWD}$ process, in which the virtual $\pi^{-}$produced dominantly along with a low energy proton at the weak vertex of $\mathrm{s} \Lambda$ is absorbed mainly on $\mathrm{I}=0$ isosinglet pn pair nucleons producing two high energy neutrons. The low energy proton is not likely to overcome the detection threshold energy. Therefore essentially it produces two high energy neutrons almost in back-to-back two-body kinematics. Therefore it tends to enhance the emission of neutrons so that its contribution will reduce the $\Gamma_{n} / \Gamma_{p}$ ratio. They predicted the contribution of the $2 \mathrm{~N}-\mathrm{NMWD}$ process as high as $0.25 \Gamma_{\Lambda}$ for ${ }_{\Lambda}^{12} C[6,7]$. This model has recently been extended further to include various exchange mesons and all NN pairs, namely not only the np pair, but also the nn and pp pair [8].

\section{Experimental Progress}

\subsection{Inclusive measurement}

The NMWD of $\Lambda$ hypernuclei has been extensively investigated in a series of experiments at the $12 \mathrm{GeV}$ proton synchrotron (PS) of the High Energy Accelerator Research Organization (KEK). The mass dependence of the total width and the proton spectra for medium mass $\Lambda$ hypernuclei were measured in the background free $\left(\pi^{+}, K^{+}\right)$reaction in the experiment E307 and reported in the references [912]. The first high quality neutron spectra of NMWD of ${ }_{\Lambda}^{12} C$ and ${ }_{\Lambda}^{89} Y$ were measured in the experiment E369 with high statistics and the careful analysis of the neutron detection efficiency for which a new modified DEMOMS code was made so that it could be applied to an array of rectangular counters [13]. The results of the modified DEMONS code are compared with the measured neutron detection efficiencies in the Fig. 1. The figure shows the neutron detection efficiencies as a function of the neutron energy calculated by the DEMONS code (lines) compared with

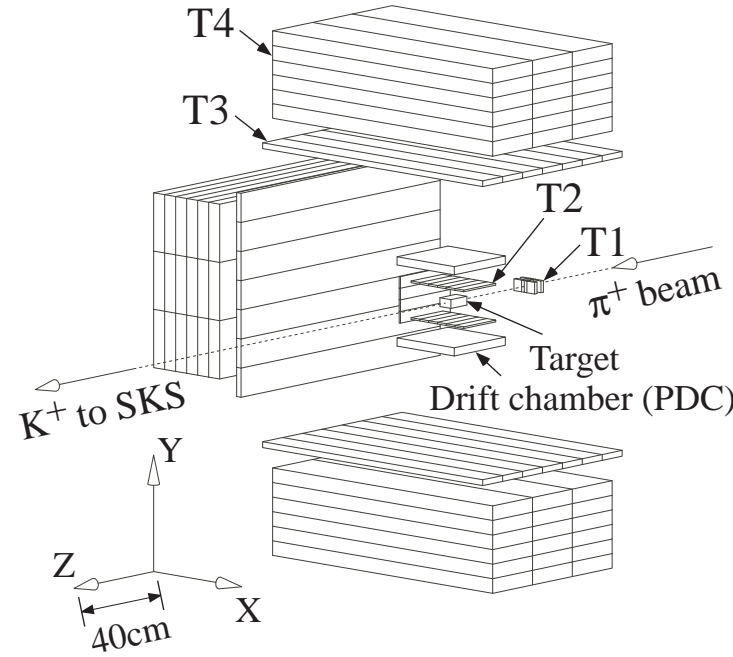

Fig. 2. The schematic view of the decay coincidence counter system. $\mathrm{T} 1$ is the time zero counter. There are three coincidence counter sets, two located at the top and bottom of the target optimized for back-to-back coincidence events and one at the side to allow the detection of non-back-to-back events. Each of the top and bottom counter sets consisted of a start-timing counter (T2), a drift chamber, a veto or stop-timing counter (T3), and neutron counter (T4) arrays. The side set was similar, except that the drift chamber was absent.

those of existing experimental data of the neutron detection efficiency measurements (data points with error bars) for various thresholds of the energy deposition in the neutron detectors. We can see that the efficiencis of the various neutron detectors are well reproduced over the wide range of the neutron energy.

The neutron spectrum made the direct comparison with that of proton possible in order to derive the experimental $\Gamma_{n} / \Gamma_{p}$ ratio. The number ratio of the emitted neutrons of E369 and protons of E307 above the common threshold energy $40 \mathrm{MeV}, 1.73 \pm 0.22$, gaved the $\Gamma_{n} / \Gamma_{p}$ ratio for the first time significantly smaller than unity, namely $0.51 \pm 0.15$ (stat. error) [6] when assumed that the NMWD consists of one-nucleon $(1 \mathrm{~N})$ induced channels, $\Lambda \mathrm{N} \rightarrow \mathrm{nN}$. The assumption was necessary in order to compensate the effect of the final state interaction (FSI), especially those due to channel cross-over which can not be cancelled in the number ratio of neutrons and protosn since there is a strong asymmetry between the neutron and proton number in the final state. The cross-over ratio was obtained by using the result of the model calculation of FSI, the intranuclear cascade calculation (INC) [13].

\subsection{Exclusive coincidence measurement}

The adoption of the direct experimental ratio of the neutron to proton number for the derivation of $\Gamma_{n} / \Gamma_{p}$ ratio was very successful cancelling the effects of the final state interaction on the neutron and the proton, and thereby removing the strong model dependence of the final state interaction. However, there still remained the inherent ambiguities due to two nucleon induced ( $2 \mathrm{~N}-)$ NMWD process, 
$19^{\text {th }}$ International IUPAP Conference on Few-Body Problems in Physics
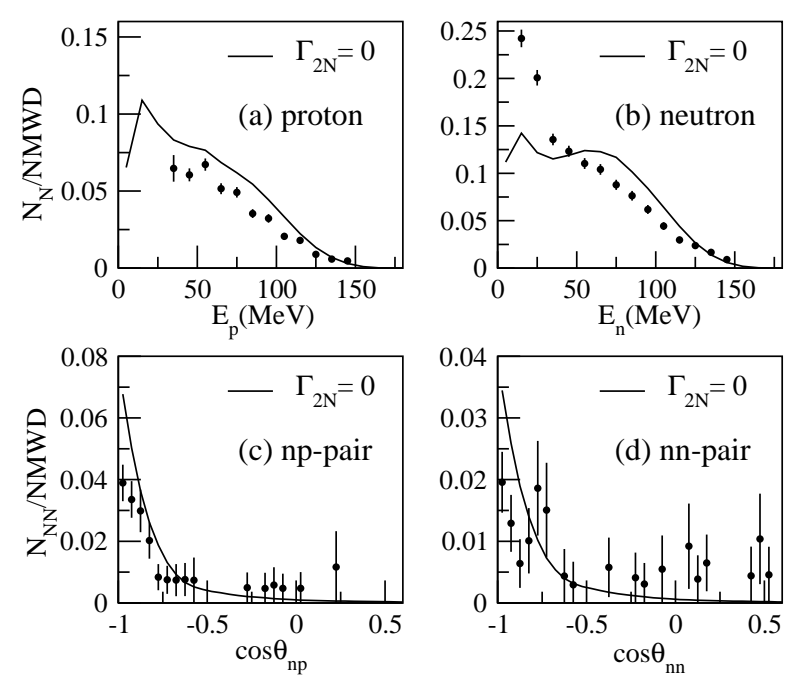

Fig. 3. The normalized proton (a) and neutron (b) spectra and the angular correlations of np (c) and nn pair (d) of NMWD of ${ }_{\Lambda}^{12} C[14-16]$ are compared with those of INC(1N) results (solid lines) for which $\Gamma_{n} / \Gamma_{p}=0.51$ was adopted.

$\Lambda \mathrm{NN} \rightarrow \mathrm{nNN}$ and some residual model dependence of the FSI calculation. Such ambiguities seem to be unavoidable in the inclusive measurement of the $\Gamma_{n} / \Gamma_{p}$ ratio. Though it has not been experimentally identified yet, the strength of the 2N-NMWD channel was predicted to be significant [7]. It was necessary to remove such ambiguities for the accurate measurement of $\Gamma_{n} / \Gamma_{p}$ ratio.

In this regards, we performed the recent high statistics measurements, E462 and E508, in which all the emitted nucleons from the NMWD of ${ }_{\Lambda}^{5} \mathrm{He}$ and ${ }_{\Lambda}^{12} \mathrm{C}$ were measured in coincidence, respectively, and the decay channels were exclusively identified. Fig. 2 shows the setup of the experiment. There are three coincidence counter sets, two located at the top and bottom of the target optimized for backto-back coincidence events and one at the side to allow the detection of non-back-to-back events. Each of the top and bottom counter sets consisted of a start-timing counter (T2), a drift chamber, a veto or stop-timing counter (T3), and neutron counter arrays (T4). The side set was similar, except that the drift chamber was absent. The details of the setup and the analysis of the experiment are refered to the reference $[14,15]$. One of the biggest systematic uncertainties was that of the neutron detection efficiency which was calculated by the modified DEMONS code after careful examination of its validity.

The spectra of emitted nucleons and the angular correlations of the two pair nucleons detected, namely the pair yields as a function of the opening angle between the momenta of the two nucleons, were reported for s-shell ${ }_{\Lambda}^{5} \mathrm{He}$ and a p-shell ${ }_{\Lambda}^{12} \mathrm{C}$ hypernuclei in the references [?,16, 15]. The results of ${ }_{\Lambda}^{12} C$ are shown in the Fig.3. The normalized proton and neutron spectra per NMWD are shown in the upper figures, (a) and (b), while the angular correlations of np and nn pair of NMWD in the lower figures, (c) and $(d)$, respectively.
Table 1. The normalized nucleon pair numbers, $N_{n p}$ and $N_{n n}$, of the NMWD of ${ }_{\Lambda}^{12} C$ in each kinematics region, back-to-back $(\cos \theta \leq-0.7$; bb) and non-back-to-back $(-0.7 \leq \cos \theta \leq 0.6$; nbb), are shown [15] and compared to those of the previous INC [7] and present INC(1N and $1 \mathrm{~N}+2 \mathrm{~N}) . N_{N N}$ is the sum, $N_{n p}+N_{n n}$.

\begin{tabular}{lllll}
\hline \hline & E508 & $\begin{array}{l}\text { INC } \\
\text { (Prev.) }\end{array}$ & $\begin{array}{l}\text { INC } \\
(1 \mathrm{~N})\end{array}$ & $\begin{array}{l}\text { INC } \\
(1 \mathrm{~N}+2 \mathrm{~N})\end{array}$ \\
\hline \hline$N_{n p}(b b)$ & $0.138 \pm 0.014$ & 0.35 & 0.231 & \\
$N_{n n}(b b)$ & $0.083 \pm 0.014$ & 0.15 & 0.118 & \\
\hline$N_{N N}(b b)$ & $0.221 \pm 0.020$ & 0.50 & 0.349 & 0.261 \\
\hline \hline$N_{n p}(n b b)$ & $0.059 \pm 0.018$ & 0.52 & 0.089 & \\
$N_{n n}(n b b)$ & $0.059 \pm 0.017$ & 0.18 & 0.046 & \\
\hline$N_{N N}(n b b)$ & $0.118 \pm 0.027$ & 0.70 & 0.135 & 0.109 \\
\hline \hline
\end{tabular}

In the experiment E508 the $\Gamma_{n} / \Gamma_{p}$ ratio was derived from the number ratio of $\mathrm{np}$ and $\mathrm{nn}$ pair satisfying the back-to-back (bb) two-body topological condition, namely $\cos \theta \leq-0.7$. The integrated pair numbers normalized to unit NMWD over each kinematics region is listed in the second column of Table 1 . The $\Gamma_{n} / \Gamma_{p}$ ratio was measured $0.51 \pm 0.14 \pm 0.04$ for ${ }_{\Lambda}^{12} C$ which are quite free from the above mentioned ambiguities [15]. These agree with the results of recent theoretical values very well and the long standing $\Gamma_{n} / \Gamma_{p}$ puzzle problem seemed to be finally solved. Naturally, the next step in the study of NMWD would be to determine the $\Gamma_{n}$ and $\Gamma_{p}$ themselves. However, in order to determine the decay width of each channel, it would be necessary to understand why the experimental $\Gamma_{n} / \Gamma_{p}$ ratios have been so high.

\section{INC and Quenching}

The upper figures of Fig. 3 show the normalized energy spectra of proton and neutron emitted per NMWD of ${ }_{\Lambda}^{12} C$ in the E508 [14] and compared with those (solid lines) of the INC (IntraNuclear Cascade) calculation for FSI which included one-nucleon induced NMWD only, which will be refered INC(1N), adopting the $\Gamma_{n} / \Gamma_{p}$ value 0.51 which is now well established. We observe that the proton yields are about 20 percent lower than that of INC calculation. If we had to determine the $\Gamma_{n} / \Gamma_{p}$ ratio out of proton measurement, we would have to compare the proton yields to that of INC varying $\Gamma_{n} / \Gamma_{p}$. Then the deficiency of proton would be compensated by increasing the $\Gamma_{n} / \Gamma_{p}$ ratio. In fact it has been the practice. In the right figure, we now see that the neutron yields are also quenched by a similar amount from that of the INC(1N) calculation. It is now clear that the quenching is universal for both proton and neutron and the old procedure compensating the proton deficiency by increasing $\Gamma_{n} / \Gamma_{p}$ ratio was not justified since the neutron yield was also quenched. We now understand that the quenching of the nucleon yields emitted in the decay was really the source of the confusion behind the long standing puzzle.

As a consequence of the quenching, the coincidence nucleon pair yields must also be quenched accordingly. 

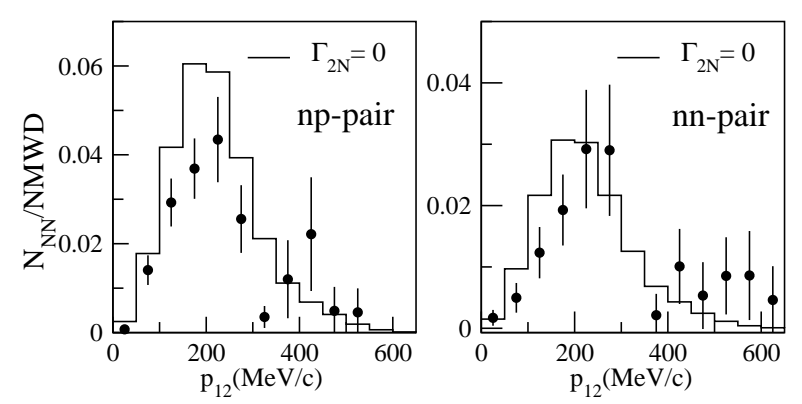

Fig. 4. The normalized momentum sum distributions of $n p$ and nn pairs of NMWD of ${ }_{\Lambda}^{12} C$ are compared with those of INC(1N) (histogram) with $\Gamma_{n} / \Gamma_{p}=0.51$.

In the lower figures of Fig. 3, np (c) and nn (d) pair angular correlations are also compared to those of INC(1N) shown in solid lines. Again we clearly observe the significant quenching. In the Table 1 the numerical nucleon pair yields, $N_{n p}$ and $N_{n n}$, per NMWD of ${ }_{\Lambda}^{12} C$ are shown for each kinematics region and compared to those of various INC calculations. The non-back-to-back (nbb) kinematics region is defined as $-0.7 \leq \cos \theta \leq 0.6$ excluding the region near $\cos \theta=1$ where are non-negligible event contribution in that single nucleon into the counter can be misidentified as a pair event due to the neutron cross-over in the counter. The measured total pair number $N_{N N}\left(=N_{n p}+N_{n n}\right)$ in the back-to-back kinematics region is quenched about 56 and 37 percent from those of the previous and present INC(1N) calculation, respectively [7].

Quenching mechanism; Then what would be the mechanism for the nucleon quenching? Two mechanisms can be considered, namely a stronger FSI strength than that used in the INC calculation and 3-body or manybody NMWD process. A stronger FSI would push the nucleon spectrum to lower energy region so that the observed quenching would be explained. However, INC calculation itself is the model of FSI which has been tested for a good reproduction of the nuclear scattering and reaction cross section. In order to reproduce the quenching, one would need a drastically stronger FSI, which would be unphysical.

\section{Momentum sum distribution ;}

Fig. 4 shows our recent result of the normalized NN pair yields of the NMWD of ${ }_{\Lambda}^{12} C$ in the momentum sum $\left(p_{12}=\left|\mathbf{p}_{1}+\mathbf{p}_{2}\right|\right)$ of the two emitted nucleons of the experiment E508 [17]. The np and nn pair distributions of the momentum sum show two groups, one at the low momentum centered around $200 \mathrm{MeV} / \mathrm{c}$ and the other at the high momentum around $500 \mathrm{MeV} / \mathrm{c}$. The events in the low momentum group mostly correspond to those in the backto-back kinematics $(-1 \leq \cos \theta \leq-0.7)$ in the angular correlations of Fig. 3, thereby cancelling the momentum of each other. The events in the high momentum group indicate that there must be the third party which carries the conjugate momentum to conserve the initial momentum. Therefore, the high momentum events directly indicate the existence of the non-two-body decay in NMWD, namely those of 3-body or many-body process. The solid lines are the prediction of the INC(1N) calculation. In the low mo- mentum region, we again observe the quenching in both np and nn pair yields. In order to reproduce the quenching of nucleons (and pair nucleons) yields, next we introduce the 3-body NMWD process, namely the two-nucleon induced $(2 \mathrm{~N}-)$ NMWD, in addition to the 1N-NMWD.

It is now clear that the INC based on 1N-NMWD overproduce nucleon and nucleon pair yields. In order to study the quenching of nucleons, we introduced the three-body NMWD process in addition to the traditional 1N-NMWD in our INC calculation code. At the moment, the threebody is 3 nucleons so that it is equivalent to 2N-NMWD. Previously Garbarino et al. reported the results of the INC calculations on the nucleon spectra and angular correlation of nucleon pair incorporating the $2 \mathrm{~N}-\mathrm{NMWD}$ with $\Gamma_{2 N}=0.2 \Gamma_{n m}$ in the reference [7]. The third column of Table 1 shows their results for the pair yields in each region, but the yields much overproduced. We reported recently that the INC incoorperated with $2 \mathrm{~N}-\mathrm{NMWD}$ can explain the quenching of nucleon yields successfully, but only with a large branching ratio of 2N-NMWD channel [18]. However, the further numerical determination of its contribution has been defered due to the uncontrolled INC model dependence.

INC calculation Next we briefly explain the ingredient of the present INC calculation code in which $2 \mathrm{~N}$ NMWD is incorporated to 1N-NMWD.

We have formulated an INC calculation based on the Monte Carlo technique. In order to establish the functionality of the INC, first we tested it for the proton inelastic, $\left(\mathrm{p}, \mathrm{p}^{\prime}\right)$ scattering and charge exchange, $(\mathrm{p}, \mathrm{n}$ ') reaction over wide dynamic ranges of mass and energy fixing the INC intrinsic nuclear shape parameters such as the radius and thickness. Then we apply these parameters to the INC calculation of FSI of the emitted nucleon in NMWD.

Nuclear structure is treated as a mixture of degenerate Fermi gas of neutrons and protons in the realistic WoodSaxon type density distribution. The nuclear potential is taken to be the sum of Fermi surface energy at the local point and the nucleon seperation energy. Therefore, there are no free parameter to adjust in the calculation. A vertex point is randomly selected according to the mean free path $\lambda=\left(\rho_{N} \sigma_{t}\right)^{-1}$ incorporating the Pauli principle. $\rho_{N}$ is the nuclear density and $\sigma_{t}$ the total NN scattering cross section adopted from those of the free NN scattering experiments [19]. The momentum distribution of the target nucleons is assumed to be those of zero temperature Fermi gas of the local density. A straight line approximation is used for the trajectory of a nucleon.

We have confirmed that the INC model calculation reproduced the experimental inelastic scattering (p,p') and the charge exchange reaction $\left(\mathrm{p}, \mathrm{n}^{\prime}\right)$ data quite well and were able to fix the INC intrinsic parameters. Overall the present simple INC model calculation reproduces the angle integrated total cross sections of inelstic and charge exchange reactions of the target nuclei from carbon to zirconium well over the wide energy range up to 160 $\mathrm{MeV}$ which covers exactly that of the emitted nucleons in NMWD.

Then we apply the INC model for the final state interactions of the emitted nucleons in the NMWD of ${ }_{\Lambda}^{12} C$. A 
$19^{\text {th }}$ International IUPAP Conference on Few-Body Problems in Physics

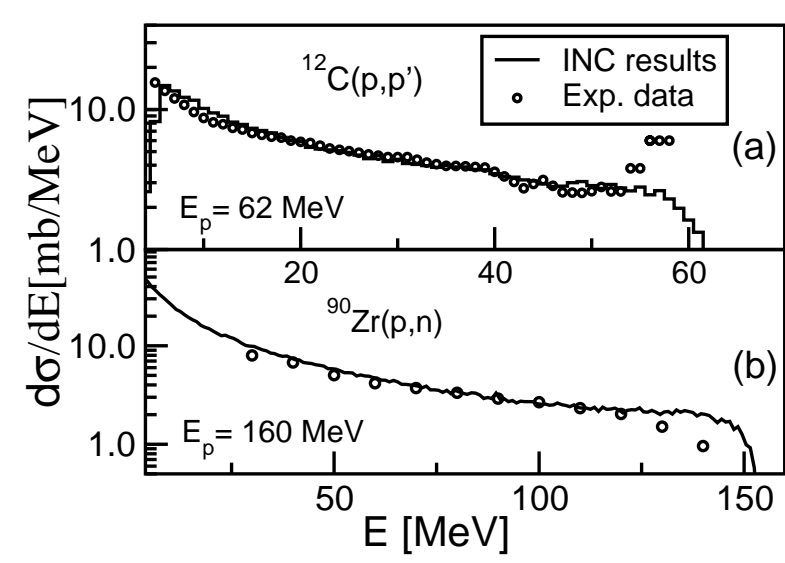

Fig. 5. The results of renormalized INC calculation (histogram) for $d \sigma / d E$ of ${ }^{12} C\left(p, p^{\prime}\right)$ at $62 \mathrm{MeV}$ and ${ }^{90} \mathrm{Zr}\left(p, n^{\prime}\right)$ at $160 \mathrm{MeV}$ are compared to the experimental data $[20,21]$

$\Lambda$ is produced at a weak interaction site by the Monte Carlo method according to the desnity and momentum distribution of 1s-state in the harmonic oscillator potential. The density and the momentum distribution of the interaction partner nucleon at the site is that of Fermi gas model. The solid lines of the Fig.1 which were compared to the experimental nucleon spectra are the results of the present INC calculation, but including only $1 \mathrm{~N}-\mathrm{NMWD}$ process.

INC calculation for $2 \mathrm{~N}$-NMWD process: In a $2 \mathrm{~N}$ NMWD process, three nucleons were produced at a vertex point with the kinematics of uniform phase space sharing. This is in somewhat different kinematics from that of the above-mentioned previous INC calculation whose kinematics was similar to that of 2-body. However, we have selected the current one considering a couple of points. First, the previous two-body like kinematics model could not reproduce the quenching of nucleons as can be seen in the fifth column of Table 1. Second, we study the effects of three-body phase space first before we introduce any particular dynamic model for 2N-NMWD.

\section{The branching ratio of 3-body NMWD}

Next we explain how we measured the branching ratio $b_{2 N}$ of the 3-body process from the quenching of the low momentum group. Since the nucleons rescattered via FSI and those of the 3-body processes share the same phase space, it is impossible to disentangle them kinematically. Therefore we extract the branching ratio by reproducing the yield quenching since it is a direct consequence of the 3-body process. Hence we need an accurate way to account for FSI. Though INC calculation is one of the most popular models for FSI, since it can be subject to a large uncertainty, we take the following approach. Instead of adopting the FSI values of INC model calculation, we varied the FSI strength of INC to fit the measured inelastic total cross section so that the FSI strength is determined from the measured data. Then we applied it to the nucleons emitted in the decay.

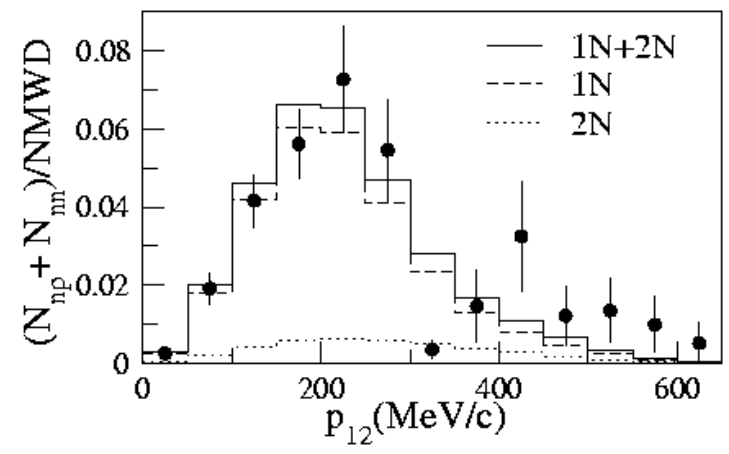

Fig. 6. The momentum sum distribution of the pair sum is compared with those of INC $(1 \mathrm{~N}+2 \mathrm{~N})$ (solid histogram) with $b_{2 N}=0.29$. The decomposed $1 \mathrm{~N}$ - (dashed one) and 2N-NMWD (dotted one) contribution also are shown.

Experimental FSI value: We first fit the experimental ${ }^{12} \mathrm{C}\left(p, p^{\prime}\right)$ inelastic scattering total cross section spectrum at $E_{p}=62 \mathrm{MeV}$ shown in Fig. 5(a) adopting the INC technique but varying the nucleon-nucleon (NN) cross section which determines the strength of FSI [20]. We introduced a renormalization fitting parameter $\alpha$ of the FSI as $\sigma_{N N}(E) \rightarrow \alpha \sigma_{N N}(E)$. We obtained $\alpha=\alpha_{0}=1.09 \pm 0.04$ by fitting the spectrum of the above reaction over a wide dynamic region from 15 to $52 \mathrm{MeV}$, except the statistical and the discrete resonance level regions. There are no adjustable parameters except for the renormalization factor. With the experimentally determined renormalization factor, $\alpha_{0}=1.09$, we were able to reproduce the total cross section distribution excellently not only for the ${ }^{12} \mathrm{C}\left(p, p^{\prime}\right)$, but also the ${ }^{90} \mathrm{Zr}(p, n)$ at $E_{p}=160 \mathrm{MeV}$ which covers a much wider dynamic range [21], as we can see in Fig. 5.

Then we applied the INC calculation with $\alpha_{0}$ for the FSI on the emitted nucleons in the decay of ${ }_{\Lambda}^{12} C$. For a 3-body process, three nucleons were produced uniformly over the 3-body phase space. The branching ratio $b_{2 N}(=$ $\left.\Gamma_{2 N} / \Gamma_{n m}\right)$ was obtained so as to reproduce the quenching in the momentum sum distribution $N_{N N}\left(p_{12}\right)\left(=N_{n p}\left(p_{12}\right)+\right.$ $\left.N_{n n}\left(p_{12}\right)\right)$ in the low momentum region $(\leq 300 \mathrm{MeV} / \mathrm{c})$. We consider the sum of nn and np pair so that it does not depend on the pair ratio of the 3-body decay model. Fig. 6 shows the pair sum distribution in the momentum sum $p_{12}$. Though the high momentum group could provide information on processes such as many nucleon-induced NMWD, the statistics are so limited that we concentrated on the low momentum group in this analysis. We obtained the branching ratio of the 3 -body process $\Lambda \mathrm{NN} \rightarrow \mathrm{nNN}, b_{2 N}=$ $\Gamma_{2 N} / \Gamma_{n m}=0.29 \pm 0.13$ by reproducing the yields at the low momentum sum region. The solid histogram shows the result of the INC calculation including 2N-NMWD with $b_{2 N}=0.29$. The dashed and dotted lines are the contributions of $1 \mathrm{~N}$ - and $2 \mathrm{~N}-\mathrm{NMWD}$ respectively. Combining this with $\Gamma_{n m}$ [22,23], we get $\Gamma_{2 N}=0.27 \pm 0.13 \Gamma_{\Lambda}$. The major errors in $\Gamma_{2 N}$ include the fitting (statistical) error (34\%), the FSI renormalization error $(13 \%)$ and that of detection efficiency $(8 \%)$.

Fig. 6 shows the INC reproductions, including the 3-body process, of the angular correlation of pair sum 

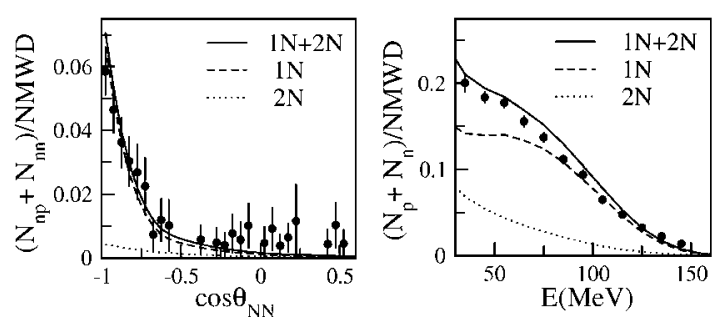

Fig. 7. The angular (left) correlation of the pair sum $N_{n p}(\theta)+$ $N_{n n}(\theta)$ and the normalized nucleon yields $N_{n}(E)+N_{p}(E)$ (right) are compared with those of $\operatorname{INC}(1 \mathrm{~N}+2 \mathrm{~N})$ (solid lines) with $b_{2 N}=0.29$. The decomposed $1 \mathrm{~N}$ - (dashed lines) and 2N-NMWD (dotted lines) contribution also are shown.

$N_{N N}(\cos \theta)$ (left) and the nucleon sum spectrum $N_{N}\left(E_{N}\right)$ (right). The solid lines are those of INC with $b_{2 N}=0.29$. Each dashed and dotted line is the contribution of $1 \mathrm{~N}$ and $2 \mathrm{~N}-\mathrm{NMWD}$, respectively. It reproduces the quenching of not only the momentum sum distribution, but also the angular correlation and the nucleon energy distribution simultaneously and successfully by including the 3-body process in addition to the two-body process. We clearly see that the low energy nucleon distribution of the right figure of Fig. 6 can be well reproduced only by including the 3 -body process. It is noted that though it requires almost 30 percent of the $2 \mathrm{~N}-\mathrm{NMWD}$ to reproduce the pair correlation, the pair yield contribution of $2 \mathrm{~N}-\mathrm{NMWD}$ in the back-to-back kinematics region is much less than 10 percent as can be seen in the left figure so that the its effect on the pair number ratio $N_{n n} / N_{n p}$ is small. Namely, the previous $\Gamma_{n} / \Gamma_{p}$ value is valid and accurate evenif the presence of the 3-body NMWD process. The present reproduction of the coincidence pair distributions and nucleon spectrum is much improved from the previous one [7]. This is the first measured $\Gamma_{2 N}$ value and its magnitude is so large that $\Gamma_{n}$ and $\Gamma_{p}$ value have to be derived by taking account of the value of $\Gamma_{2 N}$. Though the 3-body process $\Gamma_{2 N}$ is confirmed to be surprisingly large, it is in fact well consistent with the branching ratio, 0.288 , of the most recent extensive model calculation of 2N-NMWD contributions which included the effects of the complete set of exchange mesons and $\mathrm{NN}$ pairs [8].

\section{Summary}

We have shown that the reason behind the $\Gamma_{n} / \Gamma_{p}$ puzzle was the universal quenching of nucleon yields and in turn the quenching is due to the three-body process in NMWD. Reproducing the quenching of the yields of nucleons and the coincidence pair nucleons incooperating $2 \mathrm{~N}-\mathrm{NMWD}$, we have measured the branching ratio of the 3 -body NMWD process of ${ }_{\Lambda}^{12} C, 0.29 \pm 0.13$, for the first time. This is the first successful reproduction of the singles and coincidence yields of NMWD simulaneously. The contribution of the 3-body (or non-two-body) processes is so large that it should be accurately measured first before the $\Gamma_{n}$ and $\Gamma_{p}$ which are the most fundamental observables of NMWD. Now its error reaches almost 50 percent. In this regards, the next extensive coincidence experiment on NMWD is planned at the J-PARC $50 \mathrm{GeV}$ PS accelerator and the improved measurements of the decay widths of NMWD are very much awaited.

\section{Acknowledgements}

We are grateful to KEK-PS staff for the support of our experiments and for stable operation of KEK-PS. Author H.B. acknowledges supports from KOSEF(Grant No. R01-2007-000-11714-0) and KRF(Grant No. 2007-314C00069).

\section{References}

1. W. M. Alberico, G. Garbarino, Phys. Rep. 369 (2002) 1 and references therein.

2. K. Sasaki, T. Inoue and M. Oka, Nucl. Phys. A 669 (2000) 331; ibid. A678 (2000) 455.

3. D. Jido, E. Oset and J. E. Palomar, Nucl. Phys. A 694 (2001) 525

4. K. Itonaga, T. Ueda and T. Motoba, Phys. Rev. C 65 (2002) 034617.

5. W. M. Alberico et al., Phys. Lett. B 256 (1991) 134.

6. A. Ramos, E. Oset and L. L. Salcedo, Phys. Rev. C50 (1994) 2314.

7. G. Garbarino, A. Parreno and A. Ramos, Phys. Rev. Lett. 91 (2003) 112501.

8. E. Bauer and G. Garbarino, arXiv nuclth/0902:1239v1 (2009).

9. H. Bhang et al., Phys. Rev. 81 (1998) 4321.

10. H. Park et al., Phys. Rev. C 61 (2000) 054004.

11. O. Hashimoto et al., Phys. Rev. 88 (2002) 042503.

12. Y. Sato et al., Phys. Rev. C 71 (2005) 025203.

13. J.H. Kim et al., Phys. Rev. C 68 (2003) 065201.

14. S. Okada et al., Phys. Lett B 597 (2004) 249.

15. M. J. Kim et al., Phys. Lett. B 641 (2006) 28.

16. B. H. Kang, et al., Phys. Rev. Lett. 96 (2006) 062301.

17. M.J. Kim et al., Phys. Rev. Lett. 103 (2009) 182502.

18. H. Bhang et al., Eur. Phys. J. A 33 (2007) 259.

19. A. Ilinov, M. Karzarnovsky and E. Pariyev, Intermediate Energy Nuclear Physics (CRC Press, 1994).

20. F. E. Bertrand and R. W. Pelle, Phys. Rev. C 8 (1973) 1045.

21. W. Scobel et al., Phys. Rev. C 41 (1990) 2010.

22. S. Okada et al., Nucl. Phys. A 754 (2005) 178c.

23. S. Kameoka et al., Nucl. Phys. A 754 (2005) 173c. 